\title{
20 Years of RoboCup
}

\author{
Ubbo Visser ${ }^{1}$
}

Published online: 23 August 2016

(C) Springer-Verlag Berlin Heidelberg 2016

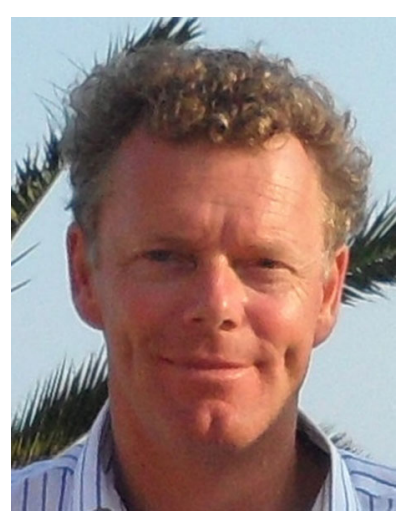

Dear readers,

Some of us will remember the first RoboCup in Germany, the 10th edition, 10 years ago, held in Bremen and widely covered in the media. We finished the 20th edition of RoboCup a few days ago, again in Germany, this time held in Leipzig from 6/30-7/4. It was a record-breaking RoboCup: more participants then ever ( $>3500$ ), more teams from more countries. The organizers, led by Prof. Gerhard Kraetzschmar from HS Rhein-Bonn Sieg, have done a fine job and made sure that this RoboCup will remain positive in peoples heads for a long time.

The editors of Künstliche Intelligenz decided to devote this special issue to RoboCup, making it the fastest special issue ever with the printed edition being on our readers desks just a few weeks after the event took place.

The speaker of the German RoboCup chapter Thomas Röfer collected the German success stories at this years RoboCup, also featuring the news for each league. The 3D soccer simulation league, e.g., introduced new automatic referee features for foul play. The humanoid league presented a newly developed humanoid robot igus, which has done very well throughout the tournament and has been

Ubbo Visser

visser@cs.miami.edu

1 Department of Computer Science, University of Miami, Coral Gables, FL 33146, USA awarded with the International Harting-Open-SourceAward, an award based on the key requirement to share the knowledge after every competition based on an open source approach. Congratulations to Sven Behnke and his team in Bonn. This year also featured a new challenge: the Amazon Picking Challenge. Stowing unsorted items from a box into shelves was one of the tasks, picking given objects from shelves was another task. A big success were the new outdoor challenge in the Standard Platform League demonstrated exiting games under natural lighting conditions (in a glass hall) on artificial turf, a challenge for both locomotion and vision with humanoid robots. The RoboCup Rescue Robot League also had a new outdoor category where injured people and material had to be transported between two locations, either following colored lines or GPS coordinates. There is a lot more to report, everyone interested can read the full summary on http://www.robo cup.de.

Our guest editors Profs Gerald Steinbauer and Alexander Ferrein did a marvelous job putting a special issue together in the given time frame while being active at the RoboCup event as well. A big "thank you" from the editors of our journal.

And now it is time to wish you a pleasant reading with the new issue of Künstliche Intelligenz - 20 years of RoboCup!

Kind regards,

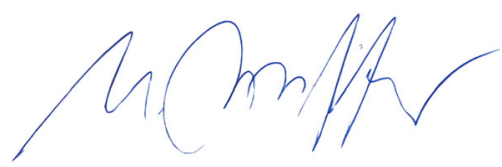




\section{Forthcoming Special Issues}

\section{Challenges for Reasoning under Uncertainty, Inconsistency, Vagueness, and Preferences}

Managing uncertainty, inconsistency, vagueness, and preferences has been extensively explored in Artificial Intelligence. During the recent years, especially with the emerging of smart services and devices, technologies for managing uncertainty, inconsistency, vagueness, and preferences to tackle the problems of dynamic, real-world scenarios have started to play a key role also in other areas, such as information systems and the (Social or Semantic) Web. These application areas have sparked another wave of strong interest into formalisms and logics for dealing with uncertainty, inconsistency, vagueness, and preferences. Important examples are fuzzy and probabilistic approaches for description logics, or rule systems for handling vagueness and uncertainty in the Semantic Web, or formalisms for handling user preferences in the context of ontological knowledge in the Social Semantic Web. While scalability of these approaches is an important issue to be addressed, also the need for combining various of these approaches with each other or more classical ways of reasoning have become obvious (hybrid reasoning under uncertainty). The aim of the special issue is to collect overview articles on important state-of-the-art formalisms and methodologies, as well as articles on emerging trends for the future.

Submissions are solicited in all areas of uncertainty, inconsistency, vagueness, and preferences handling, including (but not restricted to) the topics listed below:

- Logics of uncertain and vague reasoning, such as probability logic, possibilistic logic, and fuzzy logic.

- Reasoning with inconsistent knowledge, para-consistent logics.

- Rough sets and similarity measures.

- Formal models and semantics of uncertain data.

- Spatio-temporal uncertainty management.

- Uncertainty and inconsistency in the (Semantic) Web.

- Changes and merging of ontologies.

- Preference formalisms and user preferences.

- Data sharing and uncertainty.

- Recommender systems.

- Connections to information retrieval, data mining, and machine learning.

- Applications, in particular to mobile systems and social networks.

The special issue welcomes especially submissions that combine different approaches of uncertain reasoning, or that elaborate on connections of uncertain reasoning to other fields.
The Künstliche Intelligenz journal, which is published and indexed by Springer, supports the following lists of formats: Technical contributions, research projects, discussions, dissertation abstracts, conference reports and book reviews. If you are interested in contributing to this special issue, please contact one of the guest editors:

\section{Prof. Dr. Gabriele Kern-Isberner}

TU Dortmund

Fakultät für Informatik

Otto-Hahn-Str. 16

44221 Dortmund

E-mail: gabriele.kern-isberner@cs.uni-dortmund.de

Prof. Thomas Lukasiewicz

Professor of Computer Science

University of Oxford

Room 366, Wolfson Building

Parks Road, Oxford OX1 3QD

E-mail: thomas.lukasiewicz@cs.ox.ac.uk

\section{Landmark-based Navigation in Cognitive Systems}

The importance of landmarks in human navigation has long been recognized in multiple fields. These include areas involved in the understanding, modelling and supporting wayfinding, spatial knowledge acquisition, and place recognition. From the Psychological, Linguistic and Cognitive Neuroscience viewpoint, the perceived landmarkness of discrete objects vary among individuals. Thus, the key challenge lies in identifying those properties, which remain relevant across a wide range of individual differences, experiences, and behavioural patterns. From the Computer Science, Artificial Intelligence and Cognitive Modelling perspective, formalising these relations in a manner successfully matching the landmarks relevance for humans has proven difficult. Most recently, the increasing volume and accessibility of semantically rich geospatial data has opened new avenues for further progress in this area. The continuing collaboration between these fields is exemplified by the regular conference series on spatial information theory and geospatial science as well as multiple on-going interdisciplinary research projects.

In spite of that, technologies used to support human navigation struggle to incorporate the type of landmark information relevant for the human user. The gap between the humans and the computers understanding of what constitutes a landmark remains one of the major challenges in the development of spatial systems intuitive in use as well as in modelling navigational behaviour similar to this of a human.

This special issue integrates theoretical, experimental and computational contributions from disciplines involved in the 
study of landmark-based navigation in cognitive systems. The aim of the issue is to identify new areas for potential interdisciplinary collaboration and we invite applications focusing on, but not limited to, the following topics:

- Automatic, semi-automatic, and crowd-sourced detection of landmarks.

- Modelling of landmark-based navigation.

- Landmark knowledge acquisition and use.

- Communication of landmark-ness.

- Landmark-based approaches for indoor navigation.

- Human-computer interaction with landmark-based systems.

- Ubiquitous computing applications of the landmark concept.

The Künstliche Intelligenz Journal, which is published and indexed by Springer, supports the following lists of formats: Technical contributions, research projects, discussions, dissertation abstracts, conference reports, software, and book reviews. If you are interested in contributing to this special issue, please contact one of the guest editors as soon as possible:

\section{Prof. Dr. Angela Schwering}

Dr. Jakub Krukar

Vanessa Joy Anacta

Institute for Geoinformatics

University of Münster

48149 Münster

E-mail: schwering@uni-muenster.de;

krukar@uni-muenster.de;

v.anacta@uni-muenster.de

\section{Smart Environments-Artificial Intelligence- Human-Machine-Interaction}

Smart Environments aim to provide installations that support and enhance the abilities of humans in their regular life and possibly improve the environments themselves too, e.g., in terms of energy efficiency. Smart Environments are based on complex and distributed technical systems but they bear more challenges than the seamless composition of its components addressed in current research in technical disciplines. Setting technical challenges aside, providing intuitive interfaces to a system hidden in the environment and identifying means that allow the system automatically to provide suitable assistance for a wide range of every-day tasks involves several research questions.

Smart Environments are best described as an active field of research spanning several disciplines, but particularly related to Artificial Intelligence and Human-Machine Interaction. Since all applications penetrate our daily life with sensors, privacy becomes a central issue, too. This special issue aims at presenting a survey of the manifold AI-related research currently being performed, along with presentations of the projects and labs in which new ideas are conceived. Among the various disciplines involved, this issue addresses AI-related aspects and the interplay of $\mathrm{AI}$ and human-machine interaction, in particular AI techniques fostering new kinds of interaction, shedding some light onto questions such as:

- How can a ubiquitous system communicate its state of believe to a human?

- How can Smart Environments quickly comprehend a situation and user needs?

- How can a system adapt to users and how adaptive should it be?

- Which forms of knowledge representation help to form a shared mental model of system and user?

- How can user acceptance be measured efficiently and reliably?

- How can user acceptance be measured efficiently and reliably?

- What are social and ethical implications of Smart Environments?

- Which are the most pressing use cases? What are their specific requirements?

We welcome submissions for all kinds of articles published by KI, but especially (a) genuine research articles (max. 7 pages in print incl. authors' vita), (b) project presentations to highlight an important research question (4-6 pages), and (c) lab presentations to showcasing smart environment research installations and associated research (4-6 pages).

Please send your submissions via email directly to one of the guest editors listed below, please do not use the online submission system of the journal as otherwise submissions will not be associated with this special issue. Formatting guidelines (LATEX and Word) can be found on the journal homepage, see "author instructions" on http://www.kuenstliche-intelligenz.de.

\section{Important Dates:}

June 30, 2016: submission deadline

May 2017: journal publication

Please signal your interest in submitting a paper by sending a working title of your manuscript to the guest editors in advance.

\section{Junior prof. Dr. Diedrich Wolter}

University of Bamberg

WIAI/Smart Environments

96045 Bamberg

E-mail: diedrich.wolter@uni-bamberg.de 
Junior prof. Dr. Alexandra Kirsch

University of Tübingen

Media Informatics (Human-Computer Interaction)

Sand 14

72076 Tübingen

E-mail: alexandra.kirsch@uni-tuebingen.de

\section{Semantic Interpretation of Multi-Modal Human-Behaviour Data}

This special issue of the KI journal focusses on and emphasizes general methods and tools for activity and event-based semantic interpretation of multi-modal sensory data relevant to a range of application domains and problem contexts where interpreting human behaviour is central. The overall motivation and driving theme of the special issue pertains to AI-based methods and tools that may serve a foundational purpose toward the high-level semantic interpretation of large-scale, dynamic, multimodal sensory data, or data streams. Data-sources that may be envisaged include

- Visuo-spatial imagery

- Movement and interaction data

- Neurophysiological and other human behaviour data
Proposed foundational methods will, for instance, present the development of human-centered technologies and cognitive interaction systems aimed at assistance and empowerment, e.g. in everyday life and professional problem solving and creativity. This call particularly emphasizes systematically formalised integrative AI methods and tools (e.g., combining reasoning and learning) that enable declarative modelling, reasoning and query answering, relational learning, embodied grounding and simulation etc. Broadly, the role of declarative abstraction, knowledge representation and reasoning, and neural-symbolic learning and inference from multi-modal sensory data is highly welcome.

For details, please refer to the full Call for Papers at http://hcc.uni-bremen.de/calls/SpecialIssue-KI, or contact one of the guest editors.

\section{Prof. Dr. Mehul Bhatt}

University of Bremen, Germany

E-mail: bhatt@uni-bremen.de

http://hcc.uni-bremen.de/people-www/bhatt/

\section{Prof. Dr. Kristian Kersting}

Technical University of Dortmund, Germany

E-mail: kristian.kersting@cs.tu-dortmund.de http://www-ai.cs.uni-dortmund.de/PERSONAL/kersting. html 